\title{
Dental Management of Patients with Upcoming Liver Transplantation
}

\author{
Jordan Galabov ${ }^{1}$, Assya Krasteva $^{1}$, Evelina Odiseeva ${ }^{2}$ \\ 1. Department of Oral and Imaging diagnostic, Faculty of \\ Dental Medicine, Medical University - Sofia, Bulgaria \\ 2. Department of Anesthesiology and Intensive care, \\ Military Hospital - Sofia, Bulgaria
}

\begin{abstract}
Organ transplantation is now a widely undertaken procedure and the increase in the number of patients who have significant liver disease or having undergone liver transplant therapy will seek either preoperative or postoperative care. The successful management of oral health in organ transplantation patients necessitates close cooperation of a dental physician with the various transplant teams. This article reviews main medical problems impacting dental treatment in liver transplantation candidates: dental focus of infection; oral source of infection (periodontal disease, mucosal lesions, bacterial and fungal infections, etc); oral manifestations in transplant patients; bleeding problems; antibiotic prophylaxis and general medical conditions.
\end{abstract}

Key words: liver, transplantation, dental management, oral health

\section{Background}

Liver transplantation has become a standard treatment for end-stage liver disease and the number of recipients has grown rapidly in the last few years. There are several liver conditions that cause chronic or continuing liver inflammation, and the most common causes of end-stage liver disease are chronic viral hepatitis B and C, alcohol-related liver disease, autoimmune hepatitis, primary sclerosing cholangitis, primary biliary cirrhosis, steatohepatitis, liver disorders inherited or present at birth, and drug-induced liver damage $(1,2,3,4,5)$. In Bulgaria, Takorov et al. in a research report about 111 patients with hepatocellular carcinoma find 40,5\% of all patients suffer HCV, 29,7\% - HBV, 10,8\% - Hepatitis D virus (6). 
The compromised health and immune system of such patients place them at increased risk for systemic as well as oral infections. It has been estimated that 60 to $80 \%$ of liver transplant recipients develop an infection $(2,7)$. The cumulative effects of poor dental health, untreated dental disease, and increased susceptibility to infection suggest that dental infections could pose a significant risk for the physically debilitated transplant candidate, as well as immunosuppressed transplant recipients (7). I. Takorov reports about general diseases accompanying the compromised liver function. 62,2\% with cardiovascular disease, diabetes $-23 \%$, respiratory system disorders - $22 \%$, excretory organs malfunction - $20 \%$, obesity $-10 \%$, hypothyroidism $-4 \%$, neurologic disorders $-7 \%$. Severe accompanying disorders may be contraindicated for radical surgical manipulations (6).

A survey of organ transplant centers in USA for 1 year (2003 and 2004), found that among the 294 respondents, $9 \%$ reported that they had encountered 1 or more incidents of sepsis from a dental source in a transplant recipient (2). In addition, 34 centers (11\%) experienced 1 or more episodes of a dental infection prior to transplantation that necessitated cancellation or postponement of the surgery $(2,4)$.

\section{Aim}

This article reviews main medical problems impacting dental treatment in liver transplantation candidates.

\section{Review Results and Discussion}

\section{Oral health status in patients with liver disease}

Several studies indicate that patients with chronic hepatitis have poor oral health resulting not only in a large number of extracted teeth, caries lesions, but also involve a presence of gingival inflammation and periodontal disease $(4,8,9,10,11)$. According to research made by Guggenheimer et al. $48-55 \%$ of the patients from all age groups suffer gingivitis or any periodontal disease. VI. Panov and A. Krasteva assess the oral status of patients with chronic liver disease in Bulgaria. The authors reveal that the patients have an increased number of decayed and missing teeth, poor oral hygiene and presence of gingival inflammation resulting in elevated value of Papilla Bleeding Index (PBI), Debris Index (DI) and Calculus Index (Cl).

The same findings are mentioned by J. Guggenheimer and al. who establish that the presence of 2 or more carious teeth and/or 2 or more teeth that are mobile due to periodontal disease are indicators of severe dental disease as well as neglect of oral health (4).

A. Anand et al., and G. Novacek et al. report that oral hygiene, dental care, and periodontal parameters are worse and the number of teeth requiring treatment is higher in hepatitis patients with or without cirrhosis than in healthy subjects $(9,11)$.

P. Barbero et al. describe the dental status of 80 transplant recipients. Very poor dental hygiene is found in $85 \%$ of the patients while $45 \%$ were affected by advanced periodontal disease and $12 \%$ by chronic gingivitis. Dental caries is observed in $67 \%$ while in $20 \%$ of cases endodontic periapical lesions are found. Indicated dental care consists in $87 \%$ of cases in dental hygiene instructions, in $85 \%$ in scaling and root planing, in $63 \%$ in conservative restorations and in $40 \%$ in endodontic treatments (10). 
Paulo Sérgio da Silva Santos et al. review preliver transplant recipients. Fifty invasive dental procedures are carried out on the 33 patients. Three of them are subjected to basic periodontal treatment and 47 are subjected to multiple or simple extractions. Three surgical procedures result in postoperative complications (12).

The conclusion of the presented studies is that the monitoring of oral health by dentists before transplantation and the achievement of specific protocols of prophylaxis are helpful in the prevention of complications after transplantation in these patients. The authors mention that the main concern before the transplantation is to eliminate oral foci of infection, such as those of periapical and periodontal origin $(1,4,13)$.

\section{Managing the oral health of patients prior to liver transplantation}

Managing of the oral health before organ transplantation includes several factors that must be considered $(14,15,16,17,18,19)$ :

- Dental focus of infection

- Oral source of infection (periodontal disease, mucosal lesions, bacterial and fungal infections etc)

- Oral manifestations in transplant patients

- Bleeding problems

- Antibiotic prophylaxis

- General medical conditions

\section{Dental focus (odontogenic focus, disturbance field) of infection}

Oral focus (disturbance field) on one side may cause a disease, but on other side it can prevent or delay the treatment of another disease by blocking basic vital functions (20). The most common complication during organ transplantations is the transplanted organ rejection within the first 4 months of transplantation immunosuppressive drug therapy.

Therefore oral and dental foci of infection should be managed radically if they are present, especially in medically compromised patients $(14,15,16)$.

Paulo Sérgio da Silva Santos et al. describe the tooth extraction criteria in patients for liver transplantation: presence of residual roots; extensive carious lesions with partial crown destruction and risk of pulpal involvement; teeth with periapical lesions; semi-erupted teeth; teeth with periodontal involvement; as well as the capacity and interest of the patient in caring for and maintaining oral hygiene (21).

\section{Oral source of infection}

Miller, in "The Human Mouth as a focus of Infection" proposed a role for oral microorganisms in the development of a variety of disease in sites remote from the oral cavity (18).

Oral microbial flora is rich and unique; 300 or more different microbial species live in dental plaque, oral mucosa, gingival pockets and tongue.

Infections from dental foci are opportunistic infections from normal flora, where few bacteria dominate (aerobic and anaerobic gram-positive streptococci, anaerobic gram-negative rods e.g. Prevotella and fusobacterium species) $(19,22)$. Spreading of the infection from dental foci depends on: bacterial 
virulence; host factors; the localization of the infection (local, general, distant) and risk for distant infection is higher in patients with systemic factors e. g. blood malignancies, autoimmune diseases, and immunosuppressive treatment $(22,23)$.

Oral infections, especially periodontitis, have been found to be a risk factor for several systemic diseases such as diabetes mellitus, cardiovascular disease, respiratory disease and preterm pregnancy with low birth weight (20).

If the pre-transplant patients have periodontal disease the dental recommendation is to treat it accurately before liver transplantation. The periodontal treatment consists in scaling and root planing divided into several sections, starting from the supra-gingival area, gingival and subgingival level for cases of mild to moderate periodontitis, and prescription of $5 \%$ tranexamic acid mouthwash rinse three times a day for 7 days (21).

Candidiasis, which may arise from colonization in the oropharynx, can be a significant source of infection in liver transplant recipients. Guggenheimer identifies 17 liver transplant candidates with oral candidal lesions. All 17 patients have risk factors for candidiasis, including symptoms of xerostomia or diuretic therapy, and 15 of them have a denture or are smokers. Diabetes is another contributing factor to candidal overgrowth (4).

Although bacteria are the main cause of sialadenitis, viruses such as HCV have been implicated as causes of sialadenitis associated with xerostomia (24).

Dental treatment, including the elimination of oral and mucosal infection foci, is essential for transplant patients during both preparation and post-surgery periods; patients should be under constant dental care and undergo mycological tests, especially in the first months. Oral-source infections are a potential threat to transplant candidates because oral diseases tend to be more severe if left untreated in people who have received transplants (12).

\section{Oral manifestations in transplant patients}

Manifestations of a reduction in saliva are found in $56 \%$ of the liver transplant candidates (LTCs). The management of ascites and/or edema, frequent complications of cirrhosis, may require the use of diuretic agents that can reduce saliva production. Hyposalivation in turn, promotes the deposition and retention of dental plaque. Guggenheimer mentions that $70 \%$ of the liver transplant candidates are taking 1 or more diuretic agents, and this is significantly associated with the presence of dental plaque (4).

Liver transplant recipients have a high risk of developing squamous cell carcinoma of the upper digestive tract, which is also associated with prolonged use of immunosuppressant drugs, but it is primarily attributed to smoking $(4,12)$. Also a greater predisposition to epithelial dysplasia and carcinoma of the lip has been observed and Kaposi's sarcoma in areas of gingival hyperplasia induced by treatment with cyclosporine (25). These conditions require special care when monitoring these patients in the posttransplant period.

As a result of the long term immunosuppressive therapy the patients' immune response is reduced, which makes them more susceptible to developing infections: fungal, bacterial and viral infections. There is an 
increased prevalence of oral candidiasis and fungal infection has the highest degree of mortality rate, compared to bacterial and viral ones (25).

Several types of the human herpes virus could be found in transplant patients: cytomegalovirus is common in the first months after transplant; Epstein-Barr virus with hairy leukoplakia; herpes simplex virus, varicella-zoster virus and human herpesvirus-8. All of these viruses may display oral manifestations: Epstein-Barr virus is associated with hairy leukoplakia, herpes simplex virus is associated with aphthae, human herpesvirus-8 with Kaposi's sarcoma, varicella-zoster virus $(25,26)$.

Another side effect of the use of cyclosporine is gingival hyperplasia, which usually interferes with the patient's oral hygiene, leading to an increased susceptibility to infections, caries and periodontal disease (25).

\section{Bleeding problems}

Several factors can cause bleeding problems in organ transplant candidates, such as organ dysfunctions or their medications. Many may cause anticoagulation, and some may have a decreased platelet count. Patients with end-stage liver disease may have excessive bleeding because the liver is no longer producing sufficient amounts of clotting factors. Before treatment, assess the patient's bleeding potential with the appropriate laboratory tests and take precautions to limit bleeding (27) (Table 1).

Table 1. Blood tests and their regular values

\begin{tabular}{|l|l|}
\hline Tests & Normal values \\
\hline INR & $0,9-1,1$ \\
\hline Platelet count & $150000-400000 / \mathrm{mm}^{3}<50000 \mathrm{~mm}^{3}$ - bleeding \\
\hline Bleeding time & $1-3 \mathrm{~min}$ \\
\hline Prothrombin time & $11-15 \mathrm{sec}$ \\
\hline Thromboplastin time & $25-35 \mathrm{sec}$ \\
\hline Thrombin time & $15-20 \mathrm{sec}$ \\
\hline
\end{tabular}

In cases with patients suffering from liver disease and awaiting liver transplantation $(2,4,27)$ when dental treatment is indicated: the first step is consultation with the patient's gastroenterologist about whether antifibrinolytic drugs, vitamin $\mathrm{K}$, fresh frozen plasma, or other interventions are appropriate. The physician may decide to temporarily decrease the patient's level of anticoagulation before extensive dental surgeries. Some patients are only suitable for surgery in a hospital setting. Practical pieces of advice to the dentist are: to use aggressive suctioning techniques when performing extractions or other invasive procedures to prevent the patient from swallowing blood and manage bleeding sites with careful packing and suturing techniques. In a very small number of patients with advanced liver disease, swallowed blood may increase risk for hepatic coma. Patients with liver failure from any cause are difficult to evaluate from the standpoint of risk for oral bleeding $(4,25)$ 
Knowing the compromised general condition of the patients for liver transplantation, it is recommended:

Before any surgical dental manipulation for some laboratory tests to be performed, characterizing general status of the patients and providing the possibility to assess the condition and to decide whether additional measures are required. Usually pre-operative assessment should include bleeding time (BT), hematocrit, total blood count (TBC), differential blood count (DBC), platelet count, partial thromboplastin time (PTT) and INR (International Normalized Ratio) used especially for assessing MELD (Model for EndStage Liver Disease) value $(27,28)$.

There are various systemic methods that can be used to help compensate for the coagulopathies seen in these patients and decrease the risk of prolonged bleeding from invasive dental procedures. Vitamin $\mathrm{K}$ (at doses of $10 \mathrm{mg} \mathrm{IM}$ ) corrects hypoprothrombinemia. Fresh frozen plasma will lower the PT, and platelet transfusion addresses both quantitative and qualitative platelet defects. In the case of more significant coagulopathies the patient should be observed postoperatively in hospital $(21,28)$.

In patients with mild to moderate hemostatic impairment, (i.e., INR not greater than 3.5, or a platelet count not less than 50x109 L) careful surgical technique, including an attempt at obtaining primary wound closure and use of local hemostatic measures will usually suffice. The use of absorbable hemostatic materials improves clot formation and stability (21).

If the platelet count is less than $50.000 / \mathrm{mm}^{3}$, the patient should be transfused with fresh plasma. After treatment, it is obligatory to use local hemostatic measures (25).

\section{Antibiotic prophylaxis}

Bacterial infections like dentoalveolar abscess, dental caries and periodontal abscesses, purulent periodontis, sever gingivitis, and aggressive periodontal disease need proper culture and sensitivity tests and antibiotic prophylaxis (29). Usually selective antibiotic should be prescribed to the patient before and after dental treatment to prevent systemic infection (3).

D. Tong (30) similarly separates dental procedures into ones containing different degrees of risk of bacteremia occurrence (Table 2). 
Table 2. Dental procedures requiring antibiotic prophylaxis for patients under risk Dental procedures requiring antibiotic prophylaxis for patients under risk

- High risk category

- Tooth extraction

- Periodontal procedure that includes surgery

- Ultrasound scaling

- Root probing and planing

- Implant placing and tooth reimplantation

- Endodontic instrumentation or surgery beyond root apex
- Subgingival application of antibiotic fibers and bands

- Initial placing of orthodontic rings but not brackets

- Intraligamentary local anesthesia

- Preventive cleaning of teeth or implants with expected bleeding

Table 3. Dental procedures that do not require antibiotic prophylactics for patients under risk

\section{Dental Procedures where antibiotic prophylaxis is not needed}

- Dental restorations with or without retraction cord

- Local anesthesia (excluding intraligametary)

- Intracanal endodontic procedures after placing and build-ups

- Rubber dam pacing

- Post-operative suture removal
- Placement of orthodontic and prosthetic constructions

- Taking of dental impressions

- Teeth fluoridation

- Radiographs

- Adjustment of orthodontic constructions

- Replacement of milk teeth

Odontogenic bacteremia occurs in $100 \%$ of the cases of routine tooth extraction and in $55 \%$ of the cases following wisdom tooth extraction. In cases of periodontium and endodontic treatment - in 17 to $100 \%$ of patients. Oral bacteria cultivated from the blood circulation of patients subject to that type of surgery may include general parodontopathogens such as Actinobacillus actinomycetemcomitans, Prevotella intermedia, Porphyromonas gingivalis and Bacterioides forsythus. The gram-positive streptococcus viridans is of the greatest significance $(22,31)$.

The choice of antibiotic is better to be consulted with the patient's physician. 
It is proven that there is a chance of hematogenous spread of oral infections to the brain, heart, lungs, kidneys and knee joints. Odontogenic bacteriemia is usually short-lived. Few bacteria can be cultivated from blood ten minutes after invasive dental treatment. Patients having chronic dental infections, however, may suffer from frequent or persistent bacteremia that could become active and trigger acute and chronic inflammations in other organs $(18,19)$.

Some literary sources $(16,28)$ contain descriptions of febrile conditions of unclear etiology following transplantation surgery. The lack of precise diagnosis also results in inappropriate treatment related to the prescription of unnecessary antibiotic consumption. A possible cause to be found in the oral cavity, for example asymptomatic periodontal changes, impacted and semi-impacted wisdom teeth, is very often neglected. J. Guggenheimer (5) has expressed an opinion that it is necessary to look back to the link between oral and systemic diseases and conditions.

\section{General Medical problems}

Patients preparing to undergo organ transplantation usually take multiple medications. These include anticoagulants, beta blockers, calcium channel blockers, diuretics, and others. The dentist must be aware of the side effects of these medications, which range from xerostomia and gingival hyperplasia to orthostatic hypotension and hyperglycemia, and their interactions with drugs you might prescribe $(4,5)$.

On the other hand many medications commonly used in dental practice, including NSAIDs and some antimicrobials, are metabolized by these organs and are not removed from circulation as quickly in patients with markedly reduced kidney or liver function. Prior to dental treatment, it is recommended to decide with the patient's physician on appropriate drug selection, dosage, and administration intervals (2, $3,5)$.

Patients with end-stage organ failure usually have other major medical conditions (diabetes and/or significant pulmonary or heart disease, etc). The dentist must carefully review the patient's medical history to determine what additional treatment considerations the patient may need (20).

\section{Dental deliberation for liver transplant candidates}

Before liver transplant the dentist must be careful with the use of certain drugs $(25,29,32)$

1. Local anesthetics (lidocaine, prilocaine, mepivacaine and bupivacaine) can be used safely when the total dose of $7 \mathrm{mg} / \mathrm{kg}$ is not exceeded and when combined with epinephrine.

2. Analgesics: paracetamol should not be used on a regular basis (no more than two weeks) and must not exceed $2-2.5 \mathrm{~g}$ per $24 \mathrm{~h}$ (with $4 \mathrm{~g}$ per $24 \mathrm{~h}$ max dose). Ibuprofen and aspirin should be avoided because they have a significant hepatic metabolism. It is preferable to use morphine as a narcotic analgesic rather than meperidine and codeine, because morphine has extra-hepatic metabolism.

3. Sedatives (diazepam, lorazepam and midazolam): these drugs can be used safely if we reduce the dose and if we increase dosing intervals between medications

4. Antibiotics (clindamycin, metronidazole and vancomycin): instead of these antibiotics we should use beta-lactam antibiotics (25). 
Table 4. Dental drugs metabolized primary by the liver (32)

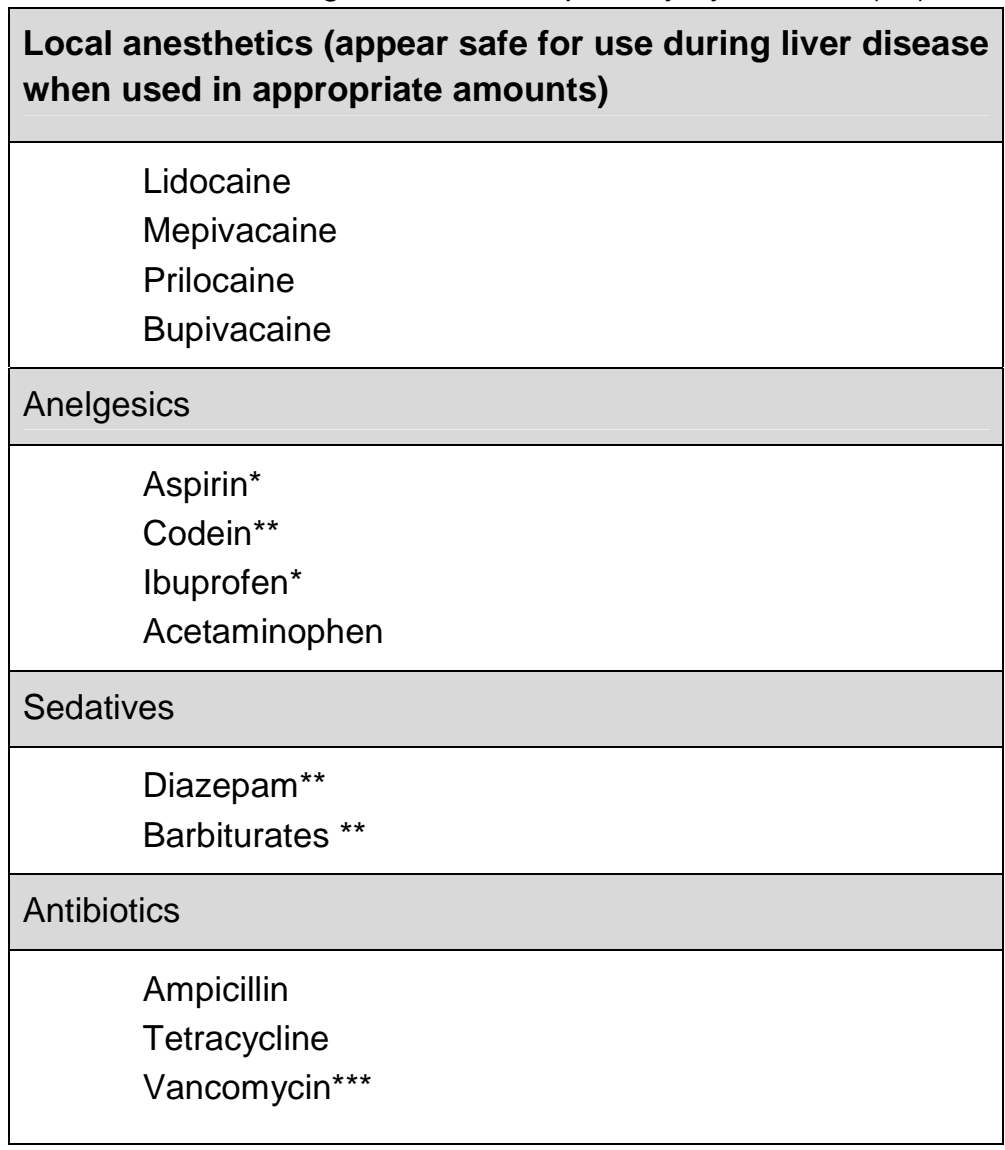

Legend:

* reduced dose or avoid if severe liver disease (active hepatitis and cirrhosis) or hemostatic abnormalities present

** highly-reduced dose or avoid if severe liver disease (active hepatitis and cirrhosis) or encephalopathy is present, or taken with alcohol

${ }^{* * *}$ avoid if severe liver disease (active hepatitis and cirrhosis) presents

\section{Conclusion}

With the increased number of transplantations worldwide, the field of progress expands rapidly, demanding new treatment approaches and evaluation of old ones. A dentist needs vast knowledge in the field of general medicine to be able to minimize adverse outcomes secondary to the provision of oral health.

\section{References}

1. Guggenheimer J, Eghtesad B, Stock DJ. Dental management of the (solid) organ transplant patient. Oral Surg Oral Med Oral Pathol OralRadiol Endod 2003;95:383-389. 
2. Guggenheimer J, Mayher D, Eghtesad B. A survey of dental care protocols among US organ transplant centers. Clin Transplant 2005;19:15-18.

3. Kamala KA, Prashant G. Oral consideration and dental management in organ transplant patient. J. Int Oral Health 2010;2-5.

4. Guggenheimer J, Eghtesad B, CloseJM, et al. Dental health status of liver transplant candidates. Liver Transpl 2007;13:2:280-286.

5. Douglas LR, Douglass JB, Sieck JO. Oral management of the patient with end-stage liver disease and the liver transplant patient. Oral Surg Oral Med Oral Pathol Oral Radiol Endod 1998;86:5564.

6. Takorov I. Contemporary surgical approaches in the treatment of the hepatocellular carcinoma. ; PhD thesis. Sofia 2014.

7. Díaz-Ortiz ML, Micó-Llorens JM, Gargallo-Albiol. Dental health in liver transplantpatients. Med Oral Patol Oral Cir Bucal 2005;10:66-72.

8. Panov VI, Krasteva A. Oral Health In Patients With Liver Diseases Journal of IMAB 2011;17:2:140-142.

9. Anand A., Pardal P, Sachdev V. Dental caries and periodontal disorders inchronic liver disease. Medical JournalArmed Forces India 2001;57:1:26-30.

10. Barbero P, Garzino Demo MG, Milanesio M, et al. The dental assessment of the patient waiting for a liver transplant. Minerva Stomatol 1996;45:10:431-439.

11. Novacek G, Plachetzky U, Potzi R, et al. Dental and periodontal disease in patients with cirrhosis - role of etiology of liver disease. J Hepatol 1995;22:5:576-582.

12. Santos P, Fernandes K, Gallottini M. Assessment and management of oral health in liver transplant candidates J. Appl. Oral Sci 2012;20:22.

13. Niederhagen B, Wolff $M$, Appel T. Location and sanitation of dental foci in liver transplantation. Transplant International 2003;16:173-178.

14. Little JW, Falace DA, Miller CS. Dental Management of the Medically Compromised Patient (8th ed.). Publisher: Mosby Inc 2012 book.

15. Hietala J, Meurman J, Grönroos L. Oral mucosal health in liver transplant recipients and controls Liver Transpl. 2014;20:1:72-80.

16. Helenius-Hietala J, Meurman JH, Höckerstedt K. Effect of the aetiology and severity of liver disease on oral health and dental treatment prior to transplantation. Transplant International 2012;25:158-165.

17. Vasanthan A, Dallal N. Periodontal treatment considerations for cell transplant and organ transplant patients. Periodontol 2000 2007;44:82-102. 
18. Miller WD. The Human Mouth as a Focus of Infection.Dental Cosmos 1891;33:9:689-706.

19. Schonfeld SE. Oral Microbial Ecology. In: Slots J, Taubman M, eds. Contemporary Oral Microbiology and Immunology. Publisher: Mosby Year 1992 book.

20. Dencheva M, Kisselova A, Krasteva A, et al. Chapter: Focal Dental Diagnostic in Patients with Replaced Renal Function - One New Method in Dentistry. In: Hemodialysis - Different Aspects. Publisher: InTech 2011.

21. Santos $P$, Pacheco M. Surgical dental treatment prior to liver transplantation. Braz J Oral Sci 2011; 10:4: 254-257.

22. Seymour R, Heasman P. Drugs, Diseases and the Periodontium. Oxford University Press 1992;46.

23. Kaufman DB, Shapiro R, Lucey MR, et al. Immunosuppression: practice and trends. Am J Transplant. 2004;4:9:38-53.

24. Hazin R, Tamimi TI, Abuzetun JY, et al. Recognizing and treating cutaneous signs of liver disease. Cleve Clin J Med 2009;76:599-606.

25. Fabuel L, Gavaldá C, Pérez G. Dental management in transplant patients. J Clin Exp Dent. 2011; $: 1: 43-52$

26. Krasteva A, Kalvachev Z, Kisselova A. Virus infections in oral medicine (In Bulgarian). Publisher: Medinform 2014 book.

27. Rada RE. Management of the dental patient on anticoagulant medication. Dent Today 2006; 5 : 58-63.

28. Ramström G, Sindet-Pedersen S, Hall G. Prevention of postsurgical bleeding in oral surgery using tranexamic acid without dose modification of oral anticoagulants. J Oral Maxillofac Surg 1993;51: 1211-1216.

29. Wynn RL, Meiller TF, Crossley HL. Drug Information Handbook for Dentistry. $12^{\text {th }}$ edition. Hudson, OH: Lexi-Comp Inc 2006:168-169.

30. Tong DC, Rothwell BR. Antibiotic prophylaxsis in dentistry. A review and practicerecommendation. JADA 2000;131:366-374.

31. Moritz A, Gutknecht N, Doertbudak O. Bacterial Reduction in Periodontal Pockets through Irradiation with a Diode Laser: A Pilot Study. Journal of Clinical Laser Medicine \& Surgery 1997;15:1:33-37.

32. Krasteva , Panov VI, Garova M et al. Hepatitis B and C in dentistry. J of IMAB 2008; 2:38-40. 


\section{Corresponding author:}

Jordan Galabov

Faculty of Dental Medicine, Department of Oral and Imaging Diagnostic 1 Sveti Georgi Sofiiski str., 1431 Sofia, Bulgaria

Email:j.galabov@gmail.com, telephone number: +359877756670 